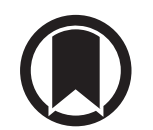

CrossMark

\title{
Metabolic consequences of obesity as an "outside in" mechanism of disease severity in asthma
}

\author{
Michael C. Peters ${ }^{1,2,3,4}$ and John V. Fahy ${ }^{1,2,3,4,5}$ \\ Affiliations: ${ }^{1}$ The Airway Clinical Research Center, University of California, San Francisco, CA, USA. ${ }^{2}$ Division \\ of Pulmonary and Critical Care Medicine, University of California, San Francisco, CA, USA. ${ }^{3}$ Cardiovascular \\ Research Institute, University of California, San Francisco, CA, USA. ${ }^{4}$ Dept of Medicine, University of \\ California, San Francisco, CA, USA. ${ }^{5}$ Sandler Center for Basic Asthma Research, University of California, San \\ Francisco, CA, USA.
}

Correspondence: John V. Fahy, Box 0130, 505 Parnassus Avenue, San Francisco, CA 94143, USA.

E-mail: john.fahy@ucsf.edu

\section{0} @ERSpublications

"Outside in" asthma: inflammatory events that drive asthma severity arise in tissue compartments outside the lung http://ow.ly/aEcZ301mcLc

The clinical features of asthma are highly varied. Most patients have relatively mild disease that is easily controlled with inhaled $\beta$-adrenergic agonists with or without low or medium doses of inhaled corticosteroids. However, a subset of patients has more severe disease, and many of these patients suffer persistent symptoms in spite of treatments that include high doses of inhaled corticosteroids. Furthermore, some asthma patients are exacerbation prone, and these patients require frequent treatment with systemic corticosteroids to control these exacerbations. Studies that have systematically examined the heterogeneity of disease severity in asthma have noted that older age and higher body weight are clinical features of patients with more severe disease $[1,2]$. In addition, it has been noted that age has a greater effect than asthma duration on risk of severe asthma [3]. These clinical features provide clues about disease mechanisms of asthma, and work is now being done to understand how ageing and obesity lead to severe asthma. For example, it has recently been shown that systemic interleukin (IL)-6 inflammation occurs in a subset of patients with asthma who are characterised by older age, obesity and severe disease (low lung function and frequent exacerbations) [4]. Notably, increases in plasma IL-6 occur in some but not all obese patients, and obese patients with high IL-6 levels have more severe asthma than obese patients with low IL-6 levels. In addition, increases in plasma IL-6 remain strongly associated with asthma severity, even when controlling for body mass index and age in regression models. This means that systemic IL-6 inflammation is an independent predictor of disease severity in asthma and that the heterogeneity of asthma severity that occurs in obese patients with asthma can be explained at least in part by the presence or absence of low-grade systemic inflammation in these patients (figure 1). Low-grade systemic inflammation in obesity is characterised by increases in systemic levels of inflammatory cytokines such as IL-6, IL-1 and tumour necrosis factor- $\alpha$, and adipokines such as leptin. These mediators originate in adipocytes and activated macrophages in adipose tissue, and contribute to mechanisms of metabolic dysfunction and metabolic syndrome in obese patients $[5,6]$. These inflammatory cytokines could alter lung function in a variety of ways, including through activation of vascular endothelial cells, airway fibroblasts, airway smooth muscle cells or airway epithelial cells [7]. In addition, IL-6 influences the differentiation of naïve T-cells into Th17-cells and could indirectly affect lung function through the effects of IL-17 [8].

Received: June 072016 | Accepted: June 132016

Support statement: The authors were support by US National Heart, Lung and Blood Institute grants HL109146 and HL107202, the Parker B. Francis Foundation, and the Sandler Center for Basic Asthma Research. Funding information for this article has been deposited with the Open Funder Registry.

Conflict of interest: Disclosures can be found alongside this article at erj.ersjournals.com

Copyright @ERS 2016 


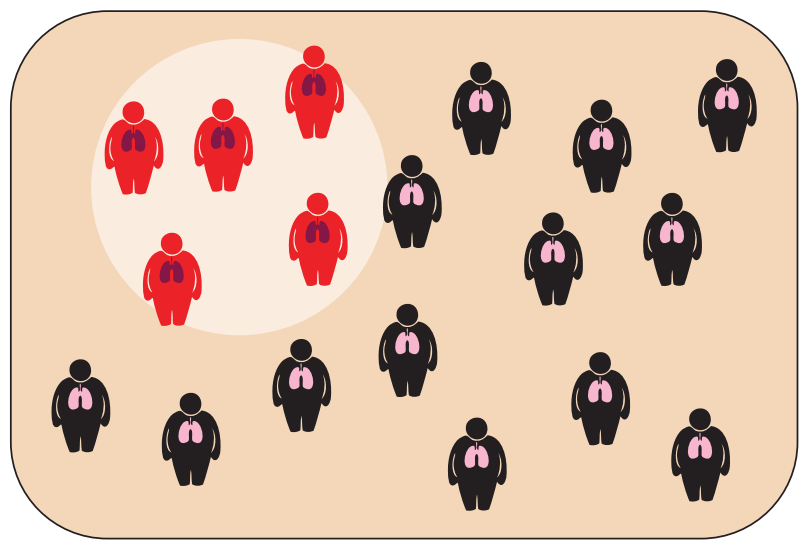

FIGURE 1 Among obese patients with asthma, only a subgroup has metabolic dysfunction. The subgroup of obese patients who have metabolic dysfunction overlaps with the subgroup with more severe asthma. Reproduced with permission from the illustrator.

Another feature of metabolic dysfunction in obese patients is insulin resistance, which results in systemic hyperinsulinaemia, and this is the focus of the paper by CARDET et al. [9] in this issue of the European Respiratory Journal. These authors investigated associations between obesity and asthma with a focus on effect modification by insulin resistance. They found that obesity was positively associated with asthma and that insulin resistance modified this association. Specifically, they found that the relationship between obesity and asthma was stronger with increasing insulin resistance tertiles, and this prompts the authors to suggest that targeting insulin resistance may represent a novel therapeutic strategy for obese patients with asthma. This finding by CARDET et al. [9] is topical because recent studies in cell culture and mouse models suggest that insulin can cause lung tissue remodelling and worsen lung function [10]. Specifically, it has been shown that insulin stimulates primary human airway smooth muscle cells to proliferate and release collagen [10]. In addition, intranasal insulin administration to mice causes peribronchial fibrosis and

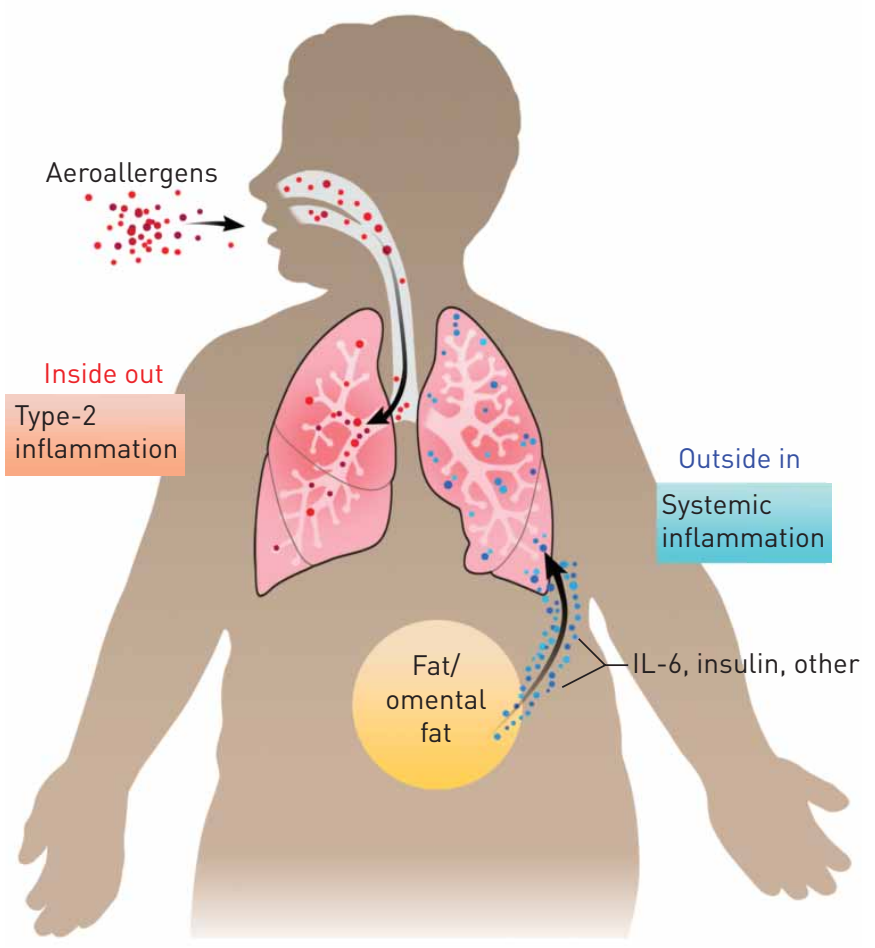

FIGURE 2 Conventional concepts of asthma pathogenesis focus on molecular pathways, such as increases in airway type-2 inflammation, that originate inside the lung, or are "inside out". New work suggests that airway dysfunction can occur from molecular dysfunction that originates outside the lung, or is "outside in". Such examples include the role of metabolic syndrome or the low-grade systemic inflammation that occurs during obesity. Reproduced with permission from the illustrator. 
airway hyperresponsiveness [10]. Thus, hyperinsulinaemia may act on insulin receptors on multiple lung cells to change airway structure and function.

Taken together, the findings that older age and obesity are risk factors for more severe asthma, coupled with data linking systemic inflammation and insulin resistance to more severe asthma, provide a new paradigm for thinking about mechanisms of disease in asthma. Current conventional concepts of asthma pathogenesis hold that disease mechanisms intrinsic to the lung are the main drivers of disease in asthma. In this "inside out" model of asthma pathogenesis the dysfunctional immune response is initiated by airway epithelial cell activation in response to inhaled triggers such as antigens or viral infections [11]. This epithelial cell activation then drives additional innate and adaptive type-2 immune response in the lung (figure 2). However, there has been little progress to date in identifying other "inside out" or intrinsic lung mechanisms of asthma and extensive analyses of lung biospecimens from patients with Th2-low asthma have not led to significant new insights about asthma pathogenesis. In contrast, there has been significant progress from studies that examine inflammatory pathways outside the lung, including IL-6 inflammation in plasma and hyperinsulinaemia. This leads us to propose an "outside in" mechanism of disease in asthma in which the inflammatory events that drive disease severity do not arise in the lung but in tissue compartments outside the lung (figure 2). In obese patients, for example, the tissue compartment is the adipose tissue. Conceptualising an outside-in mechanism of asthma severity should prompt consideration of treatment approaches for severe asthma that focus on decreasing systemic inflammation. Such treatments could include inhibiting cytokines associated with systemic inflammation (e.g. IL-6) and other treatments that are directed at improving the features of metabolic syndrome [12]. In this regard, we agree with CARDET et al. [9] that treating insulin resistance may represent a novel therapeutic strategy for obese patients with asthma.

\section{References}

1 Haldar P, Pavord ID, Shaw DE, et al. Cluster analysis and clinical asthma phenotypes. Am J Respir Crit Care Med 2008; 178: 218-224.

2 Moore WC, Meyers DA, Wenzel SE, et al. Identification of asthma phenotypes using cluster analysis in the Severe Asthma Research Program. Am J Respir Crit Care Med 2010; 181: 315-323.

3 Zein JG, Dweik RA, Comhair SA, et al. Asthma is more severe in older adults. PLoS One 2015; 10: e0133490.

4 Peters MC, McGrath KW, Hawkins GA, et al. Plasma interleukin-6 concentrations, metabolic dysfunction, and asthma severity: a cross-sectional analysis of two cohorts. Lancet Respir Med 2016 [in press DOI: 10.1016/S22132600(16)30048-0].

$5 \quad$ Kern PA, Ranganathan S, Li C, et al. Adipose tissue tumor necrosis factor and interleukin-6 expression in human obesity and insulin resistance. Am J Physiol Metab 2001; 280: E745-E751.

6 Lumeng CN, Saltiel AR. Inflammatory links between obesity and metabolic disease. J Clin Invest 2011; 121: 2111-2117.

7 Rincon M, Irvin CG. Role of IL-6 in asthma and other inflammatory pulmonary diseases. Int J Biol Sci 2012; 8 $1281-1290$.

8 Yang XO, Panopoulos AD, Nurieva R, et al. STAT3 regulates cytokine-mediated generation of inflammatory helper T cells. J Biol Chem 2007; 282: 9358-9363.

9 Cardet JC, Ash S, Kusa T, et al. Insulin resistance modifies the association between obesity and current asthma in adults. Eur Respir J 2016; 48: 403-410.

10 Singh S, Bodas M, Bhatraju NK, et al. Hyperinsulinemia adversely affects lung structure and function. Am J Physiol Lung Cell Mol Physiol 2016; 310: L837-L845.

11 Lambrecht BN, Hammad H. The airway epithelium in asthma. Nat Med 2012; 18: 684-692.

12 Grundy SM. Drug therapy of the metabolic syndrome: minimizing the emerging crisis in polypharmacy. Nat Rev Drug Discov 2006; 5: 295-309. 